\title{
New Results on the Forgotten Topological Index and Coindex
}

\author{
Akbar Jahanbani $\mathbb{D}^{1},{ }^{1}$ Maryam Atapour $\mathbb{D}^{2},{ }^{2}$ and Rana Khoeilar ${ }^{1}$ \\ ${ }^{1}$ Department of Mathematics, Azarbaijan Shahid Madani University Tabriz, Tabriz, Iran \\ ${ }^{2}$ Department of Mathematics and Computer Science, Basic Science Faculty, University of Bonab, P.O. Box 55513-95133, \\ Bonab, Iran \\ Correspondence should be addressed to Akbar Jahanbani; akbar.jahanbani92@gmail.com and Maryam Atapour; \\ maryam.atapour@gmail.com
}

Received 7 September 2021; Accepted 22 October 2021; Published 2 November 2021

Academic Editor: Ali Ahmad

Copyright (C) 2021 Akbar Jahanbani et al. This is an open access article distributed under the Creative Commons Attribution License, which permits unrestricted use, distribution, and reproduction in any medium, provided the original work is properly cited.

The $\mathscr{F}$-coindex (forgotten topological coindex) for a simple connected graph $\mathscr{G}$ is defined as the sum of the terms $\zeta_{\mathscr{G}}^{2}(y)+\zeta_{\mathscr{G}}^{2}(x)$ over all nonadjacent vertex pairs $(x, y)$ of $\mathscr{G}$, where $\zeta_{\mathscr{G}}(y)$ and $\zeta_{\mathscr{G}}(x)$ are the degrees of the vertices $y$ and $x$ in $\mathscr{G}$, respectively. The $\mathscr{F}$-index of a graph is defined as the sum of cubes of the vertex degrees of the graph. This was introduced in 1972 in the same paper where the first and second Zagreb indices were introduced to study the structure dependency of total $\pi$-electron energy. Therefore, considering the importance of the $\mathscr{F}$-index and $\mathscr{F}$-coindex, in this paper, we study these indices, and we present new bounds for the $\mathscr{F}$-index and $\mathscr{F}$-coindex.

\section{Introduction}

Suppose $\mathscr{G}$ be a simple graph with vertex set $V=V(\mathscr{G})$ and edge set $E(\mathscr{G})$. The integers $\aleph=\aleph(\mathscr{G})=|V(\mathscr{G})|$ and $\epsilon=\epsilon(\mathscr{G})=|E(\mathscr{G})|$ are the order and the size of the graph $\mathscr{G}$, respectively; we say $\mathscr{G}$ is a $(\aleph, \epsilon)$-graph. The open neighborhood of vertex $v$ is $N_{\mathscr{G}}(x)=N(x)=\{y \in V(\mathscr{G}) \mid$ $x y \epsilon E(\mathscr{G})\}$, and the degree of $v$ is $\zeta_{\mathscr{G}}(x)=|N(x)|$. We write $\Theta$ and $\delta$ for the maximum and minimum degrees of $\mathscr{G}$, respectively. A graph $\mathscr{G}$ is said to be $t$-regular if all of its vertices have degree $t$. An $(r, s)$-semiregular graph is a graph whose each vertex is of degree $s$ or $r$, and a $(k, s, t)$-triangular graph is a graph whose each vertex is of degree $k, s$, or $t$. The complement $\bar{G}$ of a graph $\mathscr{G}$ is a graph that has the same vertices as $\mathscr{G}$ and in which two vertices are adjacent if and only if they are not adjacent in $\mathscr{G}$. The number of vertex pairs $\left(x_{i}, x_{j}\right)$ in $\mathscr{G}$ such that $x_{i} x_{j} \notin E(\mathscr{G})$ is $\bar{\epsilon}=\aleph(\aleph-1) /$ $2-\epsilon(\mathscr{G})$. A pendant vertex is a vertex of degree one. The number of pendant vertices in $\mathscr{G}$ is denoted by $r=r(\mathscr{G})$. We denote by $\pi_{1}$ the minimal nonpendant vertex degree.

In $[1,2]$, the first and second Zagreb indices are defined as the following:

$$
\begin{aligned}
& M_{1}=M_{1}(\mathscr{G})=\sum_{x \in V(\mathscr{G})} \zeta_{\mathscr{G}}^{2}(x)=\sum_{x y \in E(\mathscr{G})}\left(\zeta_{\mathscr{G}}(y)+\zeta_{\mathscr{G}}(x)\right), \\
& M_{2}=M_{2}(\mathscr{G})=\sum_{x y \in E(\mathscr{G})} \zeta_{\mathscr{G}}(y) \zeta_{\mathscr{G}}(x)
\end{aligned}
$$

respectively. The first and second Zagreb coindices are defined in $[3,4]$ as

$$
\begin{aligned}
& \bar{M}_{1}(\mathscr{G})=\sum_{x y \notin E(\mathscr{G})}\left(\zeta_{\mathscr{G}}(y)+\zeta_{\mathscr{G}}(x)\right), \\
& \bar{M}_{2}(\mathscr{G})=\sum_{x y \notin E(\mathscr{G})} \zeta_{\mathscr{G}}(y) \zeta_{\mathscr{G}}(x) .
\end{aligned}
$$

Furtula and Gutman [5] defined the forgotten topological index $(\mathscr{F}$-index) as the following:

$$
\mathscr{F}=\mathscr{F}(\mathscr{G})=\sum_{x \in V(\mathscr{G})} \zeta_{\mathscr{G}}^{3}(x)=\sum_{x y \in E(\mathscr{G})}\left(\zeta_{\mathscr{G}}^{2}(y)+\zeta_{\mathscr{G}}^{2}(x)\right) .
$$

For more information on the $\mathscr{F}$-index, see [6-8]. The $\mathscr{F}$-coindex introduced in [9] is as follows: 


$$
\overline{\mathscr{F}}(\mathscr{G})=\sum_{x y \notin E(\mathscr{G})}\left(\zeta_{\mathscr{G}}^{2}(y)+\zeta_{\mathscr{G}}^{2}(x)\right) .
$$

Gutman in [10] introduced the hyper-Zagreb coindex as follows:

$$
\overline{\mathrm{HM}}(\mathscr{G})=\sum_{x y \notin E(\mathscr{G})}\left(\zeta_{\mathscr{G}}(y)+\zeta_{\mathscr{G}}(x)\right)^{2} .
$$

Usha et al. [11] introduced the redefined first Zagreb indices as the following:

$$
\operatorname{Re} Z G_{1}(\mathscr{G})=\sum_{x y \in E(\mathscr{G})} \frac{\zeta_{\mathscr{G}}(y)+\zeta_{\mathscr{G}}(x)}{\zeta_{\mathscr{G}}(y) \zeta_{\mathscr{G}}(x)} .
$$

Here, we introduce the redefined first Zagreb coindex as the following:

$$
\overline{\operatorname{Re} Z G}_{1}(\mathscr{G})=\sum_{x y \notin E(\mathscr{G})} \frac{\zeta_{\mathscr{G}}(y)+\zeta_{\mathscr{G}}(x)}{\zeta_{\mathscr{G}}(y) \zeta_{\mathscr{G}}(x)} .
$$

The authors introduced the first general Zagreb coindex in [12], and it is defined as follows:

$$
\bar{M}_{1}^{\alpha}(\mathscr{G})=\sum_{x y \notin E(\mathscr{G})}\left(\zeta_{\mathscr{G}}^{\alpha-1}(y)+\zeta_{\mathscr{G}}^{\alpha-1}(x)\right),
$$

where $\alpha \in R$. The second general Zagreb coindex was introduced in [13] and defined as follows:

$$
\bar{M}_{2}^{\alpha}(\mathscr{G})=\sum_{x y \notin E(\mathscr{G})}\left(\zeta_{\mathscr{G}}(y) \zeta_{\mathscr{G}}(x)\right)^{\alpha} .
$$

Topological indices are numerical quantity derived from a molecular graph which correlate the physicochemical properties of the molecular graph. Recently, topological indices have been studied by many researchers due to their applications in various sciences such as chemistry, physics, and electricity; see [14-16]. Among the topological indices, the first Zagreb index is one of the oldest and most applied topological indices, and for this reason, it is of great importance and has been considered by many researchers today. Furtula and Gutman in [5] recently investigated this index and named this index as "forgotten topological index" or " $F$-index" and showed that the predictive ability of this index is almost similar to that of the first Zagreb index and for the entropy and acetic factor; both of them yield correlation coefficients greater than 0.95 . Therefore, due to the importance of the $F$-index in this paper, we have decided to study this index.

In $[17,18]$, some of bounds for the general Zagreb coindices were obtained. Ranjini et al. [19] presented some of the bounds for Zagreb indices and the Zagreb coindices. In [20], some bounds were presented for the $\mathscr{F}$-index and $\mathscr{F}$-coindex. For more other bounds, see [21-23].

Given the importance of the forgotten topological index and the fact that it has recently attracted the attention of researchers and the interest of many readers, in this paper, we intend to discuss new bounds for this index.

\section{Preliminaries}

Here, we recall several published results that we will need for proof.

The following result obtained the relationship between the first Zagreb index and the maximum and minimum degrees.

Theorem 1 (see [24]). Let $\mathscr{G}$ be an $(\aleph, \epsilon)$-connected graph and $\aleph \geq 2$. Then,

$$
M_{1} \geq \frac{4 \epsilon^{2}}{\aleph}+\frac{1}{2}(\Theta-\delta)^{2},
$$

with equality if and only if $\mathscr{G}$ is isomorphic with a regular graph.

The following result comes from [18].

Theorem 2. Suppose $\mathscr{G}$ be a $(\aleph, \epsilon)$-graph and $\aleph \geq 2$. Then,

$$
M_{1} \leq \frac{4 \epsilon^{2}}{\aleph}+\frac{\aleph}{4}(\Theta-\delta)^{2},
$$

with equality if and only if $\mathscr{G}$ is isomorphic with the $t$-regular graph, $1 \leq t \leq \mathrm{N}-1$.

In [19], the authors gave the relation of the second Zagreb coindex, the maximum degree, and the first Zagreb index as follows.

Theorem 3. Let $\mathscr{G}$ be an $(\aleph, \epsilon)$-graph and maximal degree be $\Theta$. Then,

$$
\bar{M}_{2}(\mathscr{G}) \leq 2 \Theta \epsilon(\aleph-1)-\Theta M_{1}(\mathscr{G}) .
$$

Zhou and Trinajstić [25] proved the following result.

Theorem 4. Suppose $\mathscr{G}$ be a $(\aleph, \epsilon)$-graph. Then,

$$
\mathscr{F}(\mathscr{G}) \geq \frac{16 \epsilon^{3}}{\aleph^{2}}-2 M_{2}(\mathscr{G})
$$

with equality if and only if $\mathscr{G}$ is regular.

Furtula and Gutman [5] showed the following.

Theorem 5. Suppose $\mathscr{G}$ be a graph of size $\epsilon$. Then,

$$
\mathscr{F}(\mathscr{G}) \geq \frac{M_{1}(\mathscr{G})^{2}}{2 \epsilon},
$$

with equality if and only if $\mathscr{G}$ is regular.

In [26], Elumalai et al. obtained the following two results.

Theorem 6. Suppose $\mathscr{G}$ be a simple graph of order $\aleph \geq 2$. Then,

$$
\mathscr{F}(\mathscr{G}) \geq \Theta^{3}+\Theta_{2}^{3}+\Phi,
$$

with equality if and only if $\mathscr{G}$ is regular, where 


$$
\begin{aligned}
\Phi= & \frac{\left(M_{1}(\mathscr{G})-\Theta^{2}+\Theta_{2}^{2}\right)^{2}+(\aleph-2)\left(M_{1}(\mathscr{G})-\Theta^{2}+\Theta_{2}^{2}\right)}{\left(2 \epsilon-\Theta+\Theta_{2}\right)} \\
& -\left(2 \epsilon-\Theta+\Theta_{2}\right) .
\end{aligned}
$$

Theorem 7. Suppose $\mathscr{G}$ be a simple graph of order $\aleph \geq 2$. Then,

$$
\mathscr{F}(\mathscr{G}) \geq \Theta^{3}+\Theta_{2}^{3}+Y
$$

with equality if and only if $\mathscr{G}$ is regular, where

$$
Y=\frac{\left(M_{1}(\mathscr{G})-\Theta^{2}-\delta^{2}\right)^{2}+(2 \epsilon-\Theta-\delta)(I D(\mathscr{G})-1 / \Theta-1 / \delta)-(\aleph-2)^{2}}{(2 \epsilon-\Theta-\delta)} .
$$
result.

In [5], Furtula and Gutman mentioned the following

Theorem 8. Suppose $\mathscr{G}$ be a connected $(\aleph, \epsilon)$-graph and second Zagreb index $M_{2}(\mathscr{G})$. Then,

$$
\mathscr{F}(\mathscr{G}) \leq 2 M_{2}(\mathscr{G})+\epsilon(\aleph-2),
$$

with equality if and only if $\mathscr{G}$ is the star graph.

The following result was first proved in [27].

Theorem 9. Suppose $\mathscr{G}$ be a simple graph of order $\aleph$. Then,

$$
\overline{\mathscr{F}}(\mathscr{G})+\mathscr{F}(\mathscr{G})=(\aleph-1) M_{1}(\mathscr{G}) .
$$

\section{New Bounds for the $\mathscr{F}$-Coindex}

In this section, we will obtain some bounds for the $\mathscr{F}$-coindex in terms of the maximal degree, minimum degree, order, size, pendant vertex, and the first and the second Zagreb indices.

Theorem 10. Suppose $\mathscr{G}$ be a $(\aleph, \epsilon)$-graph. Then,

$$
\mathscr{F}(\overline{\mathscr{G}})+\mathscr{F}(\mathscr{G})=2(\aleph-1)^{2}(\bar{\epsilon}-2 \epsilon)+3(\aleph-1) M_{1}(\mathscr{G}) .
$$

Proof. By applying the definition of the $\mathscr{F}$-index for complement graphs, we have

$$
\begin{aligned}
\mathscr{F}(\overline{\mathscr{G}}) & =\sum_{x \in V(\mathscr{G})} \zeta_{\mathscr{G}}^{3}(x)=\sum_{x \in V(\mathscr{G})}\left(\aleph-1-\zeta_{\mathscr{G}}(x)\right)^{3}, \\
& =\sum_{x \in V(\mathscr{G})}\left((\aleph-1)^{3}-\zeta_{\mathscr{G}}^{3}(x)-3(\aleph-1) \zeta_{\mathscr{G}}^{2}(x)+3(\aleph-1)^{2} \zeta_{\mathscr{G}}(x)\right), \\
& =\sum_{x \in V(\mathscr{G})}(\aleph-1)^{3}-\sum_{x \in V(\mathscr{G})} \zeta_{\mathscr{G}}^{3}(x)+3(\aleph-1) \sum_{x \in V(\mathscr{G})} \zeta_{\mathscr{G}}^{2}(x)-3 \sum_{x \in V(\mathscr{G})}(\aleph-1)^{2} \zeta_{\mathscr{G}}(x), \\
& =\aleph(\aleph-1)^{3}-\mathscr{F}(\mathscr{G})+3(\aleph-1) M_{1}(\mathscr{G})-6 \epsilon(\aleph-1)^{2}, \\
& =2(\aleph-1)^{2}(\bar{\epsilon}-2 \epsilon)-\mathscr{F}(\mathscr{G})+3(\aleph-1) M_{1}(\mathscr{G}) .
\end{aligned}
$$

Theorem 11. Suppose $\mathscr{G}$ be a $(\aleph, \epsilon)$-graph. Then,

$$
\overline{\mathscr{F}}(\overline{\mathscr{G}})=2 \epsilon(\aleph-1)^{2}-2(\aleph-1) M_{1}(\mathscr{G})+\mathscr{F}(\mathscr{G}) .
$$

Proof. For any vertex $y \in V(\mathscr{G})$, we have $\zeta_{\overline{\mathscr{G}}}(y)=\aleph-1-\zeta_{\mathscr{G}}(y)$, and by applying the definition of the $\mathscr{F}$-coindex, we have

$$
\begin{aligned}
\overline{\mathscr{F}}(\overline{\mathscr{G}}) & =\sum_{x y \notin E(\overline{\mathscr{G}})}\left(\zeta_{\overline{\mathscr{G}}}^{2}(y)+\zeta_{\overline{\mathscr{G}}}^{2}(x)\right), \\
& =\sum_{x y \in E(\mathscr{G})}\left(\left(\aleph-1-\zeta_{\mathscr{G}}(x)\right)^{2}+\left(\aleph-1-\zeta_{\mathscr{G}}(y)\right)^{2}\right), \\
& =\sum_{x y \in E(\mathscr{G})}\left((\aleph-1)^{2}+\zeta_{\mathscr{G}}^{2}(x)-2(\aleph-1) \zeta_{\mathscr{G}}(x)+(\aleph-1)^{2}+\zeta_{\mathscr{G}}^{2}(y)-2(\aleph-1) \zeta_{\mathscr{G}}(y)\right),
\end{aligned}
$$




$$
\begin{aligned}
& =\sum_{x y \in E(\mathscr{G})}\left(2(\aleph-1)^{2}-2(\aleph-1)\left(\zeta_{\mathscr{G}}(x)+\zeta_{\mathscr{G}}(y)\right)+\left(\zeta_{\mathscr{G}}^{2}(x)+\zeta_{\mathscr{G}}^{2}(y)\right)\right), \\
& =2(\aleph-1)^{2} \epsilon-2(\aleph-1) M_{1}(\mathscr{G})+\mathscr{F}(\mathscr{G}) .
\end{aligned}
$$

Now, we give a lower bound for the $\mathscr{F}$-coindex in terms of pendant vertices.

Theorem 12. Suppose $\mathscr{G}$ be a connected graph of order $\aleph$ and $r$ pendant vertices. Then,

$$
\overline{\mathscr{F}}(\mathscr{G}) \geq 6 \curvearrowright r-4 r^{2}-7 r .
$$

Proof. It is easy to see that our result holds for $r=0$. Now, we assume that $r \geq 1$. Here, we let that $\mathscr{G}$ has exactly one pendant vertex, called $x$, and $y$ is the unique neighbor of $x$. Hence,

$$
\begin{aligned}
\overline{\mathscr{F}}(\mathscr{G}) & \geq \sum_{z \in V(\mathscr{G}) \backslash\{y, x\}}\left(\zeta_{\mathscr{G}}^{2}(z)+1\right) \geq \sum_{w \in V(\mathscr{G}) \backslash\{y, x\}} 5, \\
& =5(\aleph-2) .
\end{aligned}
$$

Here, we can let that $r \geq 2$. Each pair of pendant vertices contributes 2 to $\overline{\mathscr{F}}(\mathscr{G})$. The total contribution of pendant vertex pairs to $\overline{\mathscr{F}}(\mathscr{G})$ is $2\left(\begin{array}{l}r \\ 2\end{array}\right)$. Assume that $x$ is a pendant vertex in $\mathscr{G}$ and $y$ is its unique neighbor. Then, for any nonpendant vertex $z$ such that $w \in V(\mathscr{G}) \backslash\{y, x\}$, the contribution of vertex pairs $\{x, z\}$ to $\overline{\mathscr{F}}(\mathscr{G})$ is $1+\zeta_{\mathscr{G}}^{2}(z)$. The total contribution of such vertex pairs $\{x, z\}$ to $\overline{\mathscr{F}}(\mathscr{G})$ is $(\aleph-r-1)\left(\begin{array}{l}r \\ 1\end{array}\right)\left(1+\zeta_{\mathscr{G}}^{2}(z)\right)$. Note that $\zeta_{\mathscr{G}}(z) \geq 2$ for any nonpendant vertex $z$ in $\mathscr{G}$; therefore, we get $\overline{\mathscr{F}}(\mathscr{G}) \geq\left(\begin{array}{l}r \\ 2\end{array}\right)(1+1)+$

$(\aleph-r-1)\left(\begin{array}{l}r \\ 2\end{array}\right)(1+5)=6 \aleph r-4 r^{2}-7 r$, as desired

Theorem 13. If $\mathscr{G}$ is a $t$-regular graph of order $\aleph$, then

$$
\begin{aligned}
& \mathscr{F}(\overline{\mathscr{G}})=(\aleph-1)^{2}(\aleph(\aleph-1)-3 t \aleph)-\aleph t^{3}+3 \aleph(\aleph-1) t^{2}, \\
& \overline{\mathscr{F}}(\overline{\mathscr{G}})=\aleph t^{2}(\aleph-t-1), \\
& \overline{\mathscr{F}}(\overline{\mathscr{G}})=\aleph t\left((\aleph-1)^{2}-2 t(\aleph-1)+t^{2}\right) .
\end{aligned}
$$

Proof. We know that any $t$-regular graph of order $\aleph$ has $\aleph t / 2$ edges.

(1) By applying Theorem 10, we have

$$
\begin{aligned}
\mathscr{F}(\overline{\mathscr{G}}) & =2(\aleph-1)^{2}(\bar{\epsilon}-2 \epsilon)+3(\aleph-1) M_{1}(\mathscr{G})-\mathscr{F}(\mathscr{G}), \\
& =2(\aleph-1)^{2}\left(\frac{\aleph(\aleph-1)}{2}-\frac{3 \aleph t}{2}\right)+3(\aleph-1) n t^{2}-\aleph t^{3}, \\
& =(\aleph-1)^{2}(\aleph(\aleph-1)-3 t \aleph)-\aleph t^{3}+3 \aleph(\aleph-1) t^{2} .
\end{aligned}
$$

(2) By applying Theorem 9, we can write

$$
\mathscr{F}(\overline{\mathscr{G}})=(\aleph-1) M_{1}(\mathscr{G})-\mathscr{F}(\mathscr{G})=\aleph(\aleph-1) t^{2}-\aleph t^{3} .
$$

(3) Similarly, by applying Theorem 11, we obtain

$$
\begin{aligned}
\overline{\mathscr{F}}(\overline{\mathscr{G}}) & =2(\aleph-1)^{2} \epsilon-2(\aleph-1) M_{1}(\mathscr{G})+\mathscr{F}(\mathscr{G}), \\
& =2(\aleph-1)^{2} \frac{\aleph t}{2}-2 \aleph t^{2}(\aleph-1)+\aleph t^{3}, \\
& =\aleph t\left((\aleph-1)^{2}-2 t(\aleph-1)+t^{2}\right) .
\end{aligned}
$$

Now, we give lower and upper bounds for the $\mathscr{F}$-coindex.

Theorem 14. Suppose $\mathscr{G}$ be a connected (N, $\epsilon)$-graph, maximum degree be $\Theta$, and nonpendant minimum degree be $\pi_{1}$ and with s leaves. Then,

$$
\begin{aligned}
\overline{\mathscr{F}}(\mathscr{G}) & \leq\left(s(\aleph-s-1)\left(\Theta+\frac{1}{\Theta}\right)+\left(\frac{\Theta}{\pi_{1}}+\frac{\pi_{1}}{\Theta}\right)\left(\left(\frac{\aleph^{2}}{2}+\frac{s^{2}}{2}+\frac{3 s}{2}-\frac{\aleph}{2}-\epsilon\right)+s(s-1)-\aleph s\right)\right)(\aleph-2)^{2}, \\
& \overline{\mathscr{F}}(\mathscr{G}) \geq\left(s(\aleph-s-1)\left(\pi_{1}+\frac{1}{\pi_{1}}\right)+\left(\frac{\pi_{1}}{\Theta}+\frac{\Theta}{\pi_{1}}\right)\left(\left(\frac{\aleph^{2}}{2}+\frac{s^{2}}{2}+\frac{3 s}{2}-\frac{\aleph}{2}-\epsilon\right)+s(s-1)-\aleph s\right)\right) .
\end{aligned}
$$


Proof. It can be easily seen that the number of vertices pairs $\left(x_{i}, x_{j}\right)$ is as follows:

$$
\begin{aligned}
& X_{1}=\left\{\left(x_{i}, x_{j}\right) \mid x_{i} x_{j} \notin E(\mathscr{G})\right\},\left|X_{1}\right|=\frac{\aleph(\aleph-1)}{2}-\epsilon, \\
& X_{2}=\left\{\left(x_{i}, x_{j}\right) \mid x_{i} x_{j} \notin E(\mathscr{G}) \text { and } \zeta_{\mathscr{G}}\left(x_{i}\right)=\zeta_{\mathscr{G}}\left(x_{j}\right)=1\right\},\left|X_{2}\right|=\frac{s(s-1)}{2}, \\
& X_{3}=\left\{\left(x_{i}, x_{j}\right) \mid x_{i} x_{j} \notin E(\mathscr{G}) \zeta_{\mathscr{G}}\left(x_{i}\right)=1 \text { or } \zeta_{\mathscr{G}}\left(x_{j}\right)=1\right\},\left|X_{3}\right|=s(\aleph-s-1), \\
& X_{4}=\left\{\left(x_{i}, x_{j}\right) \mid x_{i} x_{j} \notin E(\mathscr{G}) \zeta_{\mathscr{G}}\left(x_{i}\right)>1, \text { and } \zeta_{\mathscr{G}}\left(x_{j}\right)>1\right\},\left|X_{4}\right|=\frac{\aleph(\aleph-1)}{2}-\epsilon-\frac{s(s-1)}{2}-s(\aleph-s-1) .
\end{aligned}
$$

For any vertex $x_{i}$, we have $\pi_{1} \leq \zeta_{\mathscr{G}}\left(x_{i}\right) \leq \Theta$ and $\pi_{1}^{2} \leq \zeta_{\mathscr{G}}\left(x_{i}\right) \zeta_{\mathscr{G}}\left(x_{j}\right) \leq \Theta^{2}$ for nonpendant vertices. Therefore, $x_{i} x_{j} \notin E(\mathscr{G})$.

We continue the proof with the following four cases.

Let $x_{i} x_{j} \notin E(\mathscr{G})$.

Case 1. If $\zeta_{\mathscr{G}}\left(x_{i}\right)>1$ and $\zeta_{\mathscr{G}}\left(x_{j}\right)>1$, we have

$$
\frac{\zeta_{\mathscr{G}}\left(x_{i}\right)}{\zeta_{\mathscr{G}}\left(x_{j}\right)}+\frac{\zeta_{\mathscr{G}}\left(x_{j}\right)}{\zeta_{\mathscr{G}}\left(x_{i}\right)} \leq\left(\frac{\Theta}{\pi_{1}}+\frac{\pi_{1}}{\Theta}\right) .
$$

Case 2. If $\zeta_{\mathscr{G}}\left(x_{i}\right)>1$ and $\zeta_{\mathscr{G}}\left(x_{j}\right)=1$, hence, we get

$$
\frac{\zeta_{\mathscr{G}}\left(x_{i}\right)}{\zeta_{\mathscr{G}}\left(x_{j}\right)}+\frac{\zeta_{\mathscr{G}}\left(x_{j}\right)}{\zeta_{\mathscr{G}}\left(x_{i}\right)} \leq\left(\Theta+\frac{1}{\Theta}\right) .
$$

It is clear that, for each $x_{i} x_{j} \notin E(\mathscr{G})$, $\zeta_{\mathscr{G}}\left(x_{i}\right), \zeta_{\mathscr{G}}\left(x_{j}\right) \leq \aleph-2$. Hence, by applying the definition of the $\mathscr{F}$-coindex and above facts, we can write

$$
\begin{aligned}
& \frac{\overline{\mathscr{F}}}{(\mathscr{G})}(\aleph-2)^{2}=\frac{\sum_{x_{i} x_{j} \notin E(\mathscr{G})}\left(\zeta_{\mathscr{G}}^{2}\left(x_{j}\right)+\zeta_{\mathscr{G}}^{2}\left(x_{j}\right)\right)}{(n-2)^{2}} \leq \sum_{x_{i} x_{j} \notin E(\mathscr{G})} \frac{\zeta_{\mathscr{G}}^{2}\left(x_{j}\right)+\zeta_{\mathscr{G}}^{2}\left(x_{j}\right)}{\zeta_{\mathscr{G}}\left(x_{i}\right) \zeta_{\mathscr{G}}\left(x_{j}\right)}, \\
& =\sum_{x_{i} x_{j} \notin E(\mathscr{G})}\left(\frac{\zeta_{\mathscr{G}}\left(x_{i}\right)}{\zeta_{\mathscr{G}}\left(x_{j}\right)}+\frac{\zeta_{\mathscr{G}}\left(x_{j}\right)}{\zeta_{\mathscr{G}}\left(x_{i}\right)}\right) \\
& =\sum_{\substack{i \\
x_{i} x_{j} \notin E(\mathscr{E}), \zeta_{\mathscr{G}}\left(x_{i}\right)=1, \zeta_{\mathscr{G}}\left(x_{j}\right)=1}}\left(\frac{\zeta_{\mathscr{G}}\left(x_{i}\right)}{\zeta_{\mathscr{G}}\left(x_{j}\right)}+\frac{\zeta_{\mathscr{G}}\left(x_{j}\right)}{\zeta_{\mathscr{G}}\left(x_{i}\right)}\right)+\sum_{\substack{x_{i} x_{j} \notin E(\mathscr{C}), \zeta \mathscr{G}\left(x_{i}\right)>1, \zeta_{\mathscr{G}}\left(x_{j}\right)=1}}\left(\frac{\zeta_{\mathscr{G}}\left(x_{i}\right)}{\zeta_{\mathscr{G}}\left(x_{j}\right)}+\frac{\zeta_{\mathscr{G}}\left(x_{j}\right)}{\zeta_{\mathscr{G}}\left(x_{i}\right)}\right)+\sum_{\substack{x_{i} x_{j} \notin E(\mathscr{S}), \zeta \mathscr{G}\left(x_{i}\right)>1, \zeta_{\mathscr{G}}\left(x_{j}\right)>1}}\left(\frac{\zeta_{\mathscr{G}}\left(x_{i}\right)}{\zeta_{\mathscr{G}}\left(x_{j}\right)}+\frac{\zeta_{\mathscr{G}}\left(x_{j}\right)}{\zeta_{\mathscr{G}}\left(x_{i}\right)}\right) \\
& \leq \sum_{\substack{x_{i} x_{j} \notin E(\mathscr{G}), \zeta \mathscr{G}\left(x_{i}\right)=1, \zeta \mathscr{G}\left(x_{j}\right)=1}} 2+\sum_{\substack{x_{i} x_{j} \notin E(\mathscr{G}), \zeta \mathscr{G}\left(x_{i}\right)>1, \zeta \mathscr{G}\left(x_{j}\right)=1}}\left(\Theta+\frac{1}{\Theta}\right)+\sum_{\substack{x_{i} x_{j} \notin E(\mathscr{G}), \zeta \mathscr{G}\left(x_{i}\right)>1, \zeta \mathscr{G}\left(x_{j}\right)>1}}\left(\frac{\Theta}{\pi_{1}}+\frac{\pi_{1}}{\Theta}\right), \\
& =s(s-1)+\left(\Theta+\frac{1}{\Theta}\right) s(\aleph-s-1)+\left(\frac{\Theta}{\pi_{1}}+\frac{\pi_{1}}{\Theta}\right)\left(\frac{\aleph(\aleph-1)}{2}-\epsilon-\frac{s(s-1)}{2}-s(\aleph-s-1)\right), \\
& =s(\aleph-s-1)\left(\Theta+\frac{1}{\Theta}\right)+\left(\frac{\Theta}{\pi_{1}}+\frac{\pi_{1}}{\Theta}\right)\left(\left(\frac{\aleph^{2}}{2}+\frac{s^{2}}{2}+\frac{3 s}{2}-\frac{n}{2}-\epsilon\right)+s(s-1)-\aleph s\right),
\end{aligned}
$$


as desired.

Case 3. If $\zeta_{\mathscr{G}}\left(x_{i}\right)>1$ and $\zeta_{\mathscr{G}}\left(x_{j}\right)>1$, we get

$$
\frac{\zeta_{\mathscr{G}}\left(x_{i}\right)}{\zeta_{\mathscr{G}}\left(x_{j}\right)}+\frac{\zeta_{\mathscr{G}}\left(x_{j}\right)}{\zeta_{\mathscr{G}}\left(x_{i}\right)} \geq\left(\frac{\pi_{1}}{\Theta}+\frac{\Theta}{\pi_{1}}\right) \text {. }
$$

Case 4. If $\zeta_{\mathscr{G}}\left(x_{i}\right)>1$ and $\zeta_{\mathscr{G}}\left(x_{j}\right)=1$, we get that

$$
\frac{\zeta_{\mathscr{G}}\left(x_{i}\right)}{\zeta_{\mathscr{G}}\left(x_{j}\right)}+\frac{\zeta_{\mathscr{E}}\left(x_{j}\right)}{\zeta_{\mathscr{G}}\left(x_{i}\right)} \geq\left(\pi_{1}+\frac{1}{\pi_{1}}\right) .
$$

Thus,

$$
\begin{aligned}
& \overline{\mathscr{F}}(\mathscr{G})=\sum_{x_{i} x_{j} \notin E(\mathscr{G})} \zeta_{\mathscr{G}}^{2}\left(x_{i}\right)+\zeta_{\mathscr{G}}^{2}\left(x_{j}\right) \geq \sum_{x_{i} x_{j} \notin E(\mathscr{G})} \frac{\zeta_{\mathscr{G}}^{2}\left(x_{i}\right)+\zeta_{\mathscr{G}}^{2}\left(x_{j}\right)}{\zeta_{\mathscr{G}}\left(x_{i}\right) \zeta_{\mathscr{G}}\left(x_{j}\right)} \\
& =\sum_{x_{i} x_{j} \notin E(\mathscr{G})}\left(\frac{\zeta_{\mathscr{G}}\left(x_{i}\right)}{\zeta_{\mathscr{G}}\left(x_{j}\right)}+\frac{\zeta_{\mathscr{G}}\left(x_{j}\right)}{\zeta_{\mathscr{G}}\left(x_{i}\right)}\right) \\
& =\sum_{\substack{x_{i} x_{j} \notin E(\mathscr{G}), \zeta \mathscr{G}\left(x_{i}\right)=1,}}\left(\frac{\zeta_{\mathscr{G}}\left(x_{i}\right)}{\zeta_{\mathscr{G}}\left(x_{j}\right)}+\frac{\zeta_{\mathscr{G}}\left(x_{j}\right)}{\zeta_{\mathscr{G}}\left(x_{i}\right)}\right)+\sum_{\substack{x_{i} x_{j} \notin E(\mathscr{G}), \zeta \mathscr{G}\left(x_{i}\right)>1,}}\left(\frac{\zeta_{\mathscr{G}}\left(x_{i}\right)}{\zeta_{\mathscr{G}}\left(x_{j}\right)}+\frac{\zeta_{\mathscr{G}}\left(x_{j}\right)}{\zeta_{\mathscr{G}}\left(x_{i}\right)}\right)+\sum_{\substack{x_{i} x_{j} \notin E(\mathscr{G}), \zeta \mathscr{G}\left(x_{i}\right)>1,}}\left(\frac{\zeta_{\mathscr{G}}\left(x_{i}\right)}{\zeta_{\mathscr{G}}\left(x_{j}\right)}+\frac{\zeta_{\mathscr{G}}\left(x_{j}\right)}{\zeta_{\mathscr{G}}\left(x_{i}\right)}\right) \\
& \zeta_{\mathscr{E}}\left(x_{j}\right)=1 \quad \zeta_{\mathscr{G}}\left(x_{j}\right)=1 \quad \\
& \geq \sum_{\substack{x_{i} x_{j} \notin E(\mathscr{G}), \zeta \mathscr{G}\left(x_{i}\right)=1, \zeta \mathscr{G}\left(x_{j}\right)=1}} 2+\sum_{\substack{x_{i} x_{j} \notin E(\mathscr{G}), \zeta \mathscr{G}\left(x_{i}\right)>1, \zeta \mathscr{G}\left(x_{j}\right)=1}}\left(\pi_{1}+\frac{1}{\pi_{1}}\right)+\sum_{\substack{x_{i} x_{j} \notin E(\mathscr{G}), \zeta \mathscr{(}\left(x_{i}\right)>1, \zeta \mathscr{G}\left(x_{j}\right)>1}}\left(\frac{\pi_{1}}{\Theta}+\frac{\Theta}{\pi_{1}}\right) \\
& =s(s-1)+\left(\pi_{1}+\frac{1}{\pi_{1}}\right) s(\aleph-s-1)+\left(\frac{\pi_{1}}{\Theta}+\frac{\Theta}{\pi_{1}}\right)\left(\frac{\aleph(\aleph-1)}{2}-\epsilon-\frac{s(s-1)}{2}-s(\aleph-s-1)\right), \\
& =s(\aleph-s-1)\left(\pi_{1}+\frac{1}{\pi_{1}}\right)+\left(\frac{\pi_{1}}{\Theta}+\frac{\Theta}{\pi_{1}}\right)\left(\left(\frac{\aleph^{2}}{2}+\frac{s^{2}}{2}+\frac{3 s}{2}-\frac{\aleph}{2}-\epsilon\right)+s(s-1)-\aleph s\right),
\end{aligned}
$$

and the proof is completed.

By setting $s=0$ in Theorem 14, we can obtain the following results.

Corollary 1. Suppose $\mathscr{G}$ be a connected $(\aleph, \epsilon)$-graph, maximum degree be $\Theta$, and nonpendant minimum degree be $\pi_{1}$. Then,

$$
\begin{aligned}
& \left.\overline{\mathscr{F}}(\mathscr{G}) \leq\left(\frac{\Theta}{\pi_{1}}+\frac{\pi_{1}}{\Theta}\right)\left(\frac{\aleph^{2}}{2}-\frac{\aleph}{2}-\epsilon\right)\right)(\aleph-2)^{2}, \\
& \left.\overline{\mathscr{F}}(\mathscr{G}) \geq\left(\frac{\pi_{1}}{\Theta}+\frac{\Theta}{\pi_{1}}\right)\left(\frac{\aleph^{2}}{2}-\frac{\aleph}{2}-\epsilon\right)\right) .
\end{aligned}
$$

Here, we give a lower bound for the $\mathscr{F}$-coindex.

Proposition 1. Suppose $\mathscr{G}$ be a connected graph of order $\mathrm{N}$ and size $\epsilon$. Then,

$$
\overline{\mathscr{F}}(\mathscr{G}) \geq \aleph^{2}-2 \epsilon-\aleph .
$$

Proof. By applying the definition of the $\mathscr{F}$-coindex, we have

$$
\overline{\mathscr{F}}(\mathscr{G})=\sum_{x_{i} x_{j} \notin E(\mathscr{G})}\left(\zeta_{\mathscr{G}}^{2}\left(x_{i}\right)+\zeta_{\mathscr{G}}^{2}\left(x_{j}\right)\right) \geq \sum_{x_{i} x_{j} \notin E(\mathscr{G})} 2=2\left(\frac{\aleph(\aleph-1)}{2}-\epsilon\right)=\aleph^{2}-2 \epsilon-\aleph .
$$


In the following result, we obtain the lower bound for the $\mathscr{F}$-coindex.
Proof. Clearly, for each $x_{i} x_{j} \notin E(\mathscr{G})$, we have $\max \left\{\zeta_{\mathscr{G}}\left(x_{i}\right), \zeta_{\mathscr{G}}\left(x_{j}\right)\right\} \leq \aleph-2$. It follows that

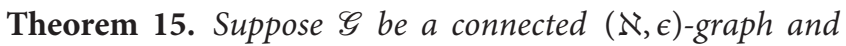
$\aleph \geq 2$. Then,

$$
\overline{\mathscr{F}}(\mathscr{G}) \geq \frac{\bar{M}_{1}^{5}(\mathscr{G})+2 \bar{M}_{2}^{2}(\mathscr{G})}{2(\aleph-2)^{2}} .
$$

$$
\begin{aligned}
2(n-2)^{2} \overline{\mathscr{F}}(\mathscr{G}) & =2(\aleph-2)^{2} \sum_{x_{i} x_{j} \notin E(\mathscr{G})}\left(\zeta_{\mathscr{G}}^{2}\left(x_{i}\right)+\zeta_{\mathscr{G}}^{2}\left(x_{j}\right)\right) \geq \sum_{x_{i} x_{j} \notin E(\mathscr{G})}\left(\zeta_{\mathscr{G}}^{2}\left(x_{i}\right)+\zeta_{\mathscr{G}}^{2}\left(x_{j}\right)\right)^{2}, \\
& =\sum_{x_{i} x_{j} \notin E(\mathscr{G})}\left(\zeta_{\mathscr{G}}^{4}\left(x_{i}\right)+\zeta_{\mathscr{G}}^{4}\left(x_{j}\right)+2 \zeta_{\mathscr{G}}^{2}\left(v_{i}\right) \zeta_{\mathscr{G}}^{2}\left(v_{j}\right)\right), \\
& =\sum_{x_{i} x_{j} \notin E(\mathscr{G})}\left(\zeta_{\mathscr{G}}^{4}\left(x_{i}\right)+\zeta_{\mathscr{G}}^{4}\left(x_{j}\right)\right)+2 \sum_{x_{i} x_{j} \notin E(\mathscr{G})}\left(\zeta_{\mathscr{G}}\left(x_{i}\right) \zeta_{\mathscr{G}}\left(x_{j}\right)\right)^{2}, \\
& =\bar{M}_{1}^{5}(\mathscr{G})+2 \bar{M}_{2}^{2}(\mathscr{G}),
\end{aligned}
$$

and the proof is completed.

$$
\overline{\mathscr{F}}(\mathscr{G}) \leq 2(\aleph-2)^{3} \overline{\operatorname{Re} Z G}_{1}(\mathscr{G})-2(\aleph-2)^{2} \bar{M}_{2}(\mathscr{G}) .
$$

Now, we give an upper bound for the $\mathscr{F}$-coindex in terms of the second Zagreb coindex and the redefined first Zagreb coindex.

Proof. By applying the definition of the $\mathscr{F}$-coindex, we have

Theorem 16. Suppose $\mathscr{G}$ be a graph of order $\aleph$. Then,

$$
\begin{aligned}
& \frac{\bar{F}(\mathscr{G})}{(n-2)^{2}}=\frac{\sum_{x_{i} x_{j} \notin E(\mathscr{G})}\left(\zeta_{\mathscr{G}}^{2}\left(x_{i}\right)+\zeta_{\mathscr{G}}^{2}\left(v_{j}\right)\right)}{(n-2)^{2}} \leq \sum_{v_{i} v_{j} \notin E(\mathscr{G})}\left(\frac{\zeta_{\mathscr{G}}\left(x_{i}\right)}{\zeta_{\mathscr{G}}\left(x_{j}\right)}+\frac{\zeta_{\mathscr{G}}\left(x_{j}\right)}{\zeta_{\mathscr{G}}\left(x_{i}\right)}\right), \\
& =\sum_{x_{i} x_{j} \notin E(\mathscr{G})}\left(\left(\frac{1}{\zeta_{\mathscr{G}}\left(x_{i}\right)}+\frac{1}{\zeta_{\mathscr{G}}\left(x_{j}\right)}\right)\left(\zeta_{\mathscr{G}}\left(x_{j}\right)+\zeta_{\mathscr{G}}\left(x_{j}\right)\right)-2 \zeta_{\mathscr{G}}\left(x_{i}\right) \zeta_{\mathscr{G}}\left(x_{j}\right)\right), \\
& =\sum_{x_{i} x_{j} \notin E(\mathscr{G})}\left(\left(\frac{1}{\zeta_{\mathscr{G}}\left(x_{i}\right)}+\frac{1}{\zeta_{\mathscr{G}}\left(x_{j}\right)}\right)\left(\zeta_{\mathscr{G}}\left(x_{j}\right)+\zeta_{\mathscr{G}}\left(x_{j}\right)\right)\right)-2 \sum_{x_{i} x_{j} \notin E(\mathscr{G})} \zeta_{\mathscr{G}}\left(x_{i}\right) \zeta_{\mathscr{G}}\left(x_{j}\right) \\
& \leq 2(n-2) \sum_{x_{i} x_{j} \notin E(\mathscr{G})}\left(\frac{1}{\zeta_{\mathscr{G}}\left(x_{i}\right)}+\frac{1}{\zeta_{\mathscr{G}}\left(x_{j}\right)}\right)-2 \sum_{x_{i} x_{j} \notin E(\mathscr{G})} \zeta_{\mathscr{G}}\left(x_{i}\right) \zeta_{\mathscr{G}}\left(x_{j}\right), \\
& =2(n-2) \sum_{x_{i} x_{j} \notin E(\mathscr{G})}\left(\frac{\zeta_{\mathscr{G}}\left(x_{i}\right)+\zeta_{\mathscr{G}}\left(x_{j}\right)}{\zeta_{\mathscr{G}}\left(x_{i}\right) \zeta_{\mathscr{G}}\left(x_{j}\right)}\right)-2 \bar{M}_{2}(\mathscr{G}), \\
& =2(\aleph-2) \overline{\operatorname{Re} Z G}_{1}(\mathscr{G})-2 \bar{M}_{2}(\mathscr{G}),
\end{aligned}
$$

which leads to the result. 
In the following results, we obtain upper bounds for the $\mathscr{F}$-coindex in terms of the first and second Zagreb indices and hyper-Zagreb coindex.

Theorem 17. Suppose $\mathscr{G}$ be a $(\aleph, \epsilon)$-graph. Then,

$$
\begin{aligned}
\overline{\mathscr{F}}(\mathscr{G})+\bar{M}_{2}(\mathscr{G}) \leq 3 \bar{\epsilon}(\aleph-2)^{2}, & (46) \quad \begin{array}{l}
\text { Proof. By applying the definitions of the } \mathscr{F} \text {-coindex and } \\
\text { second Zagreb coindex, we can write }
\end{array} \\
\overline{\mathscr{F}}(\mathscr{G})+\bar{M}_{1}(\mathscr{G}) \leq 2 \bar{\epsilon}\left(\aleph^{2}-3 \aleph+2\right), & (47) \\
\overline{\mathscr{F}}(\mathscr{G})+\bar{M}_{2}(\mathscr{G}) & =\sum_{x_{i} x_{j} \notin E(\mathscr{G})}\left(\zeta_{\mathscr{G}}^{2}\left(x_{i}\right)+\zeta_{\mathscr{G}}^{2}\left(x_{j}\right)+\zeta_{\mathscr{G}}\left(x_{i}\right) \zeta_{\mathscr{G}}\left(x_{j}\right)\right) \leq \sum_{x_{i} x_{j} \notin E(\mathscr{G})}\left((\aleph-2)^{2}+(\aleph-2)^{2}+(\aleph-2)^{2}\right), \\
& =3 \bar{\epsilon}(\aleph-2)^{2} .
\end{aligned}
$$

$$
\overline{\mathscr{F}}(\mathscr{G})+\overline{H M}(\mathscr{G}) \leq 6 \bar{\epsilon}(\aleph-2)^{2} .
$$

The equalities hold if and only if $\mathscr{G} \cong K_{\aleph}$ or $\mathscr{G}$ is $(\aleph-2)$-regular or $(\aleph-2, \aleph-1)$-semiregular.

Likewise, we have

$$
\begin{aligned}
\overline{\mathscr{F}}(\mathscr{G})+\bar{M}_{1}(\mathscr{G}) & =\sum_{x_{i} x_{j} \notin E(\mathscr{G})}\left(\zeta_{\mathscr{G}}^{2}\left(x_{i}\right)+\zeta_{\mathscr{G}}^{2}\left(x_{j}\right)+\zeta_{\mathscr{G}}\left(x_{i}\right)+\zeta_{\mathscr{G}}\left(x_{j}\right)\right) \leq \sum_{x_{i} x_{j} \notin E(\mathscr{G})}\left((\aleph-2)^{2}+(\aleph-2)^{2}+2(\aleph-2)\right), \\
& =2 \bar{\epsilon}\left(\aleph^{2}-3 \aleph+2\right),
\end{aligned}
$$

and

$$
\begin{aligned}
\overline{\mathscr{F}}(\mathscr{G})+\overline{H M}(\mathscr{G}) & =\sum_{x_{i} x_{j} \notin E(\mathscr{G})}\left(\zeta_{\mathscr{G}}^{2}\left(x_{i}\right)+\zeta_{\mathscr{G}}^{2}\left(x_{j}\right)+\left(\zeta_{\mathscr{G}}\left(x_{i}\right)+\zeta_{\mathscr{G}}\left(x_{j}\right)\right)^{2}\right), \\
& =\sum_{x_{i} x_{j} \notin E(\mathscr{G})}\left(\zeta_{\mathscr{G}}^{2}\left(x_{i}\right)+\zeta_{\mathscr{G}}^{2}\left(x_{j}\right)+\zeta_{\mathscr{G}}^{2}\left(x_{i}\right)+\zeta_{\mathscr{G}}^{2}\left(x_{j}\right)+2 \zeta_{\mathscr{G}}\left(x_{i}\right) \zeta_{\mathscr{G}}\left(x_{j}\right)\right), \\
& =\sum_{x_{i} x_{j} \notin E(\mathscr{G})}\left(2 \zeta_{\mathscr{G}}^{2}\left(x_{i}\right)+2 \zeta_{\mathscr{G}}^{2}\left(x_{j}\right)+2 \zeta_{\mathscr{G}}\left(x_{i}\right) \zeta_{\mathscr{G}}\left(x_{j}\right)\right) \leq \sum_{x_{i} x_{j} \notin E(\mathscr{G})}\left(4(\aleph-2)^{2}+2(\aleph-2)^{2}\right), \\
& =6 \bar{\epsilon}(\aleph-2)^{2} .
\end{aligned}
$$

The equalities hold in (46)-(48) if and only if $\zeta_{\mathscr{G}}\left(x_{i}\right)=\zeta_{\mathscr{G}}\left(x_{j}\right)=\aleph-2$, for each $x_{i} x_{j} \notin E(\mathscr{G})$. This implies that each vertex of $\mathscr{G}$ has degree $\aleph-1$ or $\aleph-2$; that is, $\mathscr{G} \cong$ $K_{\aleph}$ or $\mathscr{G}$ is $(\aleph-2)$-regular or $(\aleph-2, \aleph-1)$-semiregular. result.

By using Theorems $1,8,10$, and 9 , we get the following

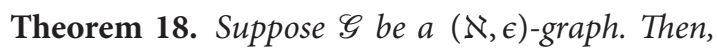

$$
\begin{array}{r}
\mathscr{F}(\overline{\mathscr{G}}) \geq 2(\aleph-1)^{2}(\bar{\epsilon}-2 \epsilon)+3(\aleph-1)\left(\frac{4 \epsilon^{2}}{\aleph}+\frac{1}{2}(\Theta-\delta)^{2}\right)-\left(2 M_{2}(\mathscr{G})+\epsilon(\aleph-2)\right), \\
\overline{\mathscr{F}}(\mathscr{G}) \geq(\aleph-1)\left(\frac{4 \epsilon^{2}}{\aleph}+\frac{1}{2}(\Theta-\delta)^{2}\right)-\left(2 M_{2}(\mathscr{G})+\epsilon(\aleph-2)\right) .
\end{array}
$$


The following results were obtained by combining Theorems 1, 4, 5, 6, and 11 .
Theorem 19. Suppose $\mathscr{G}$ be a $(\aleph, \epsilon)$-graph. Then,

$$
\begin{aligned}
& \overline{\mathscr{F}}(\overline{\mathscr{G}}) \geq 2 \epsilon(\aleph-1)^{2}-2(\aleph-1)\left(\left(\frac{4 \epsilon^{2}}{\aleph}+\frac{1}{2}(\Theta-\delta)^{2}\right)\right)+\left(\frac{16 \epsilon^{3}}{\aleph^{2}}-2 M_{2}(\mathscr{G})\right), \\
& \overline{\mathscr{F}}(\overline{\mathscr{G}}) \geq 2 \epsilon(\aleph-1)^{2}-2(\aleph-1)\left(\left(\frac{4 \epsilon^{2}}{\aleph}+\frac{1}{2}(\Theta-\delta)^{2}\right)\right)+\left(\frac{M_{1}(\mathscr{G})^{2}}{2 \epsilon}\right), \\
& \overline{\mathscr{F}}(\overline{\mathscr{G}}) \geq 2 \epsilon(\aleph-1)^{2}-2(\aleph-1)\left(\left(\frac{4 \epsilon^{2}}{\aleph}+\frac{1}{2}(\Theta-\delta)^{2}\right)\right)+\left(\Theta^{3}+\Theta_{2}^{3}+\Phi\right) .
\end{aligned}
$$

The following results were obtained by combining Theorems 2, 4, 10, and 9.

$$
\begin{aligned}
& \mathscr{F}(\overline{\mathscr{G}}) \leq 2(\aleph-1)^{2}(\bar{\epsilon}-2 \epsilon)+3(\aleph-1)\left(\frac{4 \epsilon^{2}}{\aleph}+\frac{\aleph}{4}(\Theta-\delta)^{2}\right)-\left(\frac{16 \epsilon^{3}}{\aleph^{2}}-2 M_{2}(\mathscr{G})\right), \\
& \overline{\mathscr{F}}(\mathscr{G}) \leq(\aleph-1)\left(\frac{4 \epsilon^{2}}{\aleph}+\frac{\aleph}{4}(\Theta-\delta)^{2}\right)-\left(\frac{16 \epsilon^{3}}{\aleph^{2}}-2 M_{2}(\mathscr{G})\right) .
\end{aligned}
$$

By applying Theorems 2, 8, and 11, we can obtain the following theorem.

Theorem 21. Suppose $\mathscr{G}$ be a $(\aleph, \epsilon)$-graph. Then,

$$
\begin{aligned}
\overline{\mathscr{F}}(\overline{\mathscr{G}}) \leq & 2 \epsilon(\aleph-1)^{2}-2(\aleph-1)\left(\left(\frac{4 \epsilon^{2}}{\aleph}+\frac{\aleph}{4}(\Theta-\delta)^{2}\right)\right) \\
& +\left(2 M_{2}(\mathscr{G})+\epsilon(\aleph-2)\right) .
\end{aligned}
$$

Theorem 20. Suppose $\mathscr{G}$ be a $(\aleph, \epsilon)$-graph. Then,

$$
\begin{aligned}
& \mathscr{F}(\overline{\mathscr{G}}) \leq 2(\aleph-1)^{2}(\bar{\epsilon}-2 \epsilon)+3(\aleph-1)\left(\frac{4 \epsilon^{2}}{\aleph}+\frac{\aleph}{4}(\Theta-\delta)^{2}\right)-\left(\frac{M_{1}(\mathscr{G})^{2}}{2 \epsilon}\right), \\
& \mathscr{F}(\mathscr{G}) \leq(\aleph-1)\left(\frac{4 \epsilon^{2}}{\aleph}+\frac{\aleph}{4}(\Theta-\delta)^{2}\right)-\left(\frac{M_{1}(\mathscr{G})^{2}}{2 \epsilon}\right) .
\end{aligned}
$$


The following results were obtained by combining Theorem 23. Suppose $\mathscr{G}$ be a $(\aleph, \epsilon)$-graph. Then, Theorems 7, 10, and 9.

$$
\begin{aligned}
& \mathscr{F}(\overline{\mathscr{G}}) \leq 2(\aleph-1)^{2}(\bar{\epsilon}-2 \epsilon)+3(\aleph-1)\left(\frac{4 \epsilon^{2}}{\aleph}+\frac{\aleph}{4}(\Theta-\delta)^{2}\right)-\left(\Theta^{3}+\Theta_{2}^{3}+Y\right), \\
& \overline{\mathscr{F}}(\mathscr{G}) \leq(\aleph-1)\left(\frac{4 \epsilon^{2}}{\aleph}+\frac{\aleph}{4}(\Theta-\delta)^{2}\right)-\left(\Theta^{3}+\Theta_{2}^{3}+Y\right) .
\end{aligned}
$$

\section{Conclusion}

In this paper, we investigate the relationship between the $\mathscr{F}$-coindex and the other topological coindices, such as the first and second Zagreb coindices, the hyper-Zagreb coindex, and the redefined first Zagreb coindex. However, there are still open and challenging problems for researchers, for example, the problem on the relationship among the $\mathscr{F}$-coindex and GA-coindex, harmonic coindex, Randić coindex, etc.

\section{Data Availability}

The data involved in the examples of our manuscript are included within the article.

\section{Disclosure}

The authors would like to declare that the work described was original research that has not been published previously [28].

\section{Conflicts of Interest}

The authors declare that they have no conflicts of interest.

\section{References}

[1] A. T. Balaban, I. Motoc, D. Bonchev, and O. Mekenyan, "Topological indices for structure-activity correlations," Steric effects in drug design, vol. 114, pp. 21-55, 1983.

[2] I. Gutman, B. Ruščić, N. Trinajstić, and C. F. Wilcox, "Graph theory and molecular orbitals. XII. Acyclic polyenes," The Journal of Chemical Physics, vol. 62, no. 9, pp. 3399-3405, 1975.

[3] P. S. Ranjini and V. Lokesha, "The smarandache-zagreb indices on the three graph operators," International Journal of Mathematical Combinatorics, vol. 3, pp. 1-10, 2010.

[4] P. S. Ranjini, V. Lokesha, and I. N. Cangül, "On the Zagreb indices of the line graphs of the subdivision graphs," Applied Mathematics and Computation, vol. 218, no. 3, pp. 699-702, 2011.

[5] B. Furtula and I. Gutman, "A forgotten topological index," Journal of Mathematical Chemistry, vol. 53, no. 4, pp. 11841190, 2015.

[6] Z. Du, A. Jahanbai, and S. M. Sheikholeslami, "Relationships between Randić index and other topological indices," Communications in Combinatorics and Optimization, vol. 6, pp. 137-154, 2021.
[7] I. Gutman and N. Trinajstić, "Graph theory and molecular orbitals. Total $\varphi$-electron energy of alternant hydrocarbons," Chemical Physics Letters, vol. 17, no. 4, pp. 535-538, 1972.

[8] J. Varghese Kureethara, A. Asok, and I. Naci Cangul, "Inverse problem for the forgotten and the hyper Zagreb indices of trees," Communications in Combinatorics and Optimization, vol. 7, 2021.

[9] N. De, S. M. A. Nayeem, and A. Pal, The F्F-Co-Index of Some Graph Operations, Springer, vol. 5New York, NY, USA, , 2016.

[10] I. Gutman, "On hyper-Zagreb index and co-index," Bulletin (Académie serbe des sciences et des arts. Classe des sciences mathématiques et naturelles. Sciences mathématiques), vol. 150, pp. 1-8, 2017.

[11] A. Usha, P. S. Ranjini, and V. Lokesha, "Zagreb coindices, augmented Zagreb index, redefined Zagreb indices and their polynomials for phenylene and hexagonal squeeze," in Proceedings of the International Congress in Honour of Dr. Ravi. P. Agarwal, Uludag University, Bursa, Turkey, June 2014.

[12] M. Berhe Belay and C. Wang, "The first general Zagreb coindex of graph operations," Applied Mathematics and Nonlinear Sciences, vol. 5, no. 2, pp. 109-120, 2020.

[13] H. Aram and N. Dehgardi, "Reformulated F-index of graph operations," Communications in Combinatorics and Optimization, vol. 2, pp. 87-98, 2017.

[14] M. F. Nadeem, M. Imran, H. M. Afzal Siddiqui, M. Azeem, A. Khalil, and Y. Ali, "Topological aspects of metal-organic structure with the help of underlying networks," Arabian Journal of Chemistry, vol. 14, no. 6, Article ID 103157, 2021.

[15] H. M. A. Siddiqui, S. Baby, M. F. Nadeem, and M. K. Shafiq, "Bounds of some degree based indices of lexicographic product of some connected graphs," Polycyclic Aromatic Compounds, vol. 2020, Article ID 1852266, 13 pages, 2020.

[16] T. Vetrík and S. Balachandran, "General multiplicative Zagreb indices of graphs with given clique number," Opuscula Mathematica, vol. 39, no. 3, pp. 433-446, 2019.

[17] M. Matejić, E. Milovanović, I. Milovanović, and R. Khoeilar, "A note on the first Zagreb index and co-index of graphs," Communications in Combinatorics and Optimization, vol. 6, pp. 41-51, 2021.

[18] E. Milovanovic and I. Milovanovic, "Sharp bounds for the first Zagreb index and first Zagreb coindex," Miskolc Mathematical Notes, vol. 16, no. 2, pp. 1017-1024, 2015.

[19] P. S. Ranjini, V. Lokesha, A. R. Bindusree, and M. Phani Raju, "New bounds on Zagreb indices and the Zagreb co-indices," Boletim da Sociedade Paranaense de Matemática, vol. 31, pp. 51-55, 2013.

[20] J. B. Liu, M. M. Matejić, E. I. Milovanović, and I. Z. Milovanović, "Some new inequalities for the forgotten topological index and co-index of graphs, MATCH Commun," MATCH Communications in Mathematical and in Computer, vol. 84, pp. 719-738, 2020. 
[21] R. Amin and S. M. A. Nayeem, "Extremal F-index of a graph with k cut edges," Matematicki Vesnik, vol. 72, pp. 146-153, 2020.

[22] D. Maji and G. Ghorai, "Computing F-index, coindex and Zagreb polynomials of the kth generalized transformation graphs," Heliyon, vol. 6, no. 12, Article ID e05781, 2020.

[23] D. Sarala, H. Deng, C. Natarajan, and S. K. Ayyaswamy, "F index of graphs based on four new operations related to the strong product," AKCE International Journal of Graphs and Combinatorics, vol. 17, no. 1, pp. 25-37, 2020.

[24] C. S. Edwards, "The largest vertex degree sum for a triangle in a graph," Bulletin of the London Mathematical Society, vol. 9, no. 2, pp. 203-208, 1977.

[25] B. Zhou and N. Trinajstić, "On general sum-connectivity index," Journal of Mathematical Chemistry, vol. 47, no. 1, pp. 210-218, 2010.

[26] S. Elumalai, T. Mansour, and M. A. Rostami, "On the bounds of the forgotten topological index," Turkish Journal of Mathematics, vol. 41, pp. 1687-1702, 2017.

[27] N. De, "F-index and co-index of some derived graphs," 2016, https://arxiv.org/abs/1610.02175.

[28] T. Došlić, T. Réti, and D. Vukičević, "On the vertex degree indices of connected graphs," Chemical Physics Letters, vol. 512, pp. 283-286, 2011. 